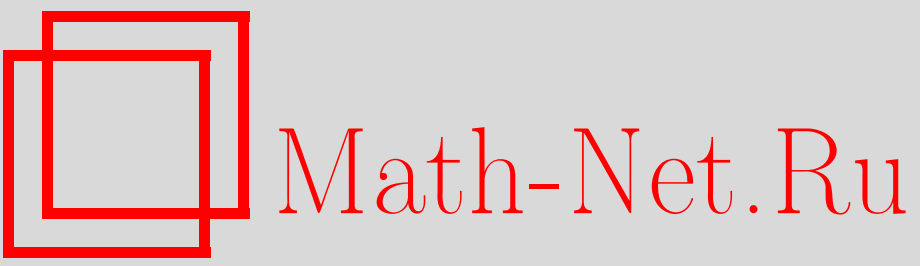

Е. А. Иванов, А. О. Сутулин, Различные типы $N=(4,4)$ мультиплетов с твистом в $N=(2,2)$ суперпространстве, TM $\Phi, 2005$, том 145 , номер $1,66-86$

DOI: https://doi.org/10.4213/tmf1881

Использование Общероссийского математического портала Math-Net.Ru подразумевает, что вы прочитали и согласны с пользовательским соглашением http: //www.mathnet.ru/rus/agreement

Параметры загрузки:

IP: 54.162 .27 .143

26 апреля 2023 г., 16:07:17 
ТЕОРЕТИЧЕСКАЯ

И МАТЕМАТИЧЕСКАЯ

ФИЗИКА

Том 145, № 1

октябрь, 2005

(C) 2005 г.

Е. А. Иванов* , А.О. Сутулин*

\title{
РАЗЛИЧНЫЕ ТИПЫ $N=(4,4)$ МУЛЬТИПЛЕТОВ С ТВИСТОМ В $N=(2,2)$ СУПЕРПРОСТРАНСТВЕ
}

\begin{abstract}
Дано описание четырех различных типов $N=(4,4)$ супермультиплетов с твистом в двумерном $N=(2,2)$ суперпространстве $\mathbb{R}^{(1,1 \mid 2,2)}$. Рассмотренные мультиплеты представляются парой суперполей - кирального суперполя и кирального суперполя с твистом - и отличаются законами преобразований относительно дополнительной скрытой $N=(2,2)$ суперсимметрии. Для каждого типа $N=(4,4)$ мультиплета с твистом рассмотрены сигма-модельные $N=(2,2)$ суперполевые лагранжианы, которые суть действительные функции, подчиненные дифференциальным связям, следующим из неявной $N=(2,2)$ суперсимметрии. Показано, что общее сигма-модельное действие, включающее различные $N=(4,4)$ мультиплеты с твистом и инвариантное относительно $N=(4,4)$ суперсимметрии, распадается в сумму сигма-модельных действий для отдельных мультиплетов. Показано, что только мультиплеты, принадлежащие самодуальной паре, могут взаимодействовать через смешанные массовые члены.
\end{abstract}

Ключевые слова: суперсимметричные сигма-модели, супермультиплеты с твистом, гармоническое суперпространство.

\section{1. ВВЕДЕНИЕ}

Важный класс двумерных суперсимметричных сигма-моделей образуют $N=(2,2)$ и $N=(4,4)$ суперсимметричные сигма-модели, бозонные многообразия которых обладают кручением и двумя независимыми взаимно коммутируюшими левыми и правыми комплексными структурами [1]-[5]. Такие модели и их частные случаи ВЗНВ типа на групповых пространствах (модели Весса-Зумино-Новикова-Виттена) используются для описания нетривиальных фоновых многообразий в $4 D$-суперструнах [6]-[8], а также имеют приложения в теории двумерных черных дыр в струнном подходе [9], [10]. Явно суперсимметричные формулировки $N=(2,2)$ моделей как в терминах $N=(2,2)$ киральных суперполей и киральных суперполей с твистом, так и в терминах $N=(2,2)$ “полукиральных" суперполей изучались в работах [2], [11]. Для $N=(4,4)$ моделей с коммутирующими комплексными структурами существуют также явно суперсиммет-

* Объединенный институт ядерных исследований, Дубна, Московская обл., Россия. E-mail: eivanov@thsun1.jinr.ru, sutulin@thsun1.jinr.ru 
ричные формулировки вне массовой оболочки в проективном [7], [9], [12] и обычном [13], [14] $N=(4,4), 2 D$ суперпространствах. Основным объектом в этих формулировках является $N=(4,4)$ мультиплет с твистом. В двумерном $N=(2,2)$ суперпространстве он представляется парой суперполей: киральным суперполем и киральным суперполем с твистом.

С другой стороны, наиболее адекватное описание теорий с расширенной суперсимметрией, в частности четырехмерных $N=2$ теорий, достигается в подходе гармонического суперпространства [15]. Двумерные $N=(4,4)$ суперсимметричные сигма-модели, которые получаются размерной редукцией из четырехмерных $N=2$ сигма-моделей, образуют подкласс в классе обших $N=(4,4), 2 D$ сигма-моделей. Действительно, их бозонные многообразия являются гиперкэлеровыми, или кватернионными кэлеровыми, т.е. многообразиями без кручения и с одним набором комплексных структур. Для описания двумерных суперсимметричных теорий с кручением оказывается необходимым привлечь более обший тип гармонического суперпространства, чем тот, который был введен и с успехом использован в четырехмерном случае. Этим новым видом гармонического суперпространства является $S U(2) \times S U(2)$ гармоническое суперпространство (бигармоническое суперпространство), определенное в работе [16]. Его характерная черта - наличие двух наборов гармонических переменных, связанных с двумя взаимно коммутируюшими группами автоморфизмов $S U(2)$ в левом и правом координатных секторах. В работе [16] было показано, как описать один тип $N=(4,4)$ мультиплета с твистом в $S U(2) \times S U(2)$ гармоническом суперпространстве, и было представлено наиболее общее действие вне массовой оболочки для этого мультиплета в виде интеграла по аналитическому подпространству полного суперпространства. Это действие отвечает общей $N=(4,4)$ суперсимметричной сигма-модели с кручением и взаимно коммутирующими наборами левых и правых комплексных структур ${ }^{1)}$. В подходе $S U(2) \times S U(2)$ гармонического суперпространства был впоследствии построен более обший класс сигма-модельных действий вне массовой оболочки, а именно действий с кручением и с некоммутирующими левыми и правыми комплексными структурами [18].

Во многих предыдущих случаях общие сигма-модельные действия с $N=(4,4)$ суперсимметрией строились для фиксированного типа мультиплетов с твистом, а именно для мультиплетов, обладаюших одинаковыми трансформационными свойствами по отношению к группе автоморфизмов $S O(4)_{\mathrm{L}} \times S O(4)_{\mathrm{R}}$ двумерной $N=(4,4)$ супералгебры Пуанкаре. При описании в $N=(2,2)$ суперпространстве это соответствует тому, что каждая пара кирального суперполя и кирального суперполя с твистом, образуюшая $N=(4,4)$ мультиплет с твистом, обладает одними и теми же трансформационными свойствами относительно дополнительной (неявной) $N=(2,2)$ суперсимметрии. С другой стороны, как будет показано в данной работе, дополнительная суперсимметрия может быть по-разному реализована на различных таких парах. Ранее было обнаружено [19]-[21], что имеется четыре сушественно различных типа $N=(4,4)$ мультиплетов с твистом (с точностью до дополнительного отражения, связанного с пространствен-

\footnotetext{
1) Некоторые другие $N=(4,4)$ мультиплеты вне массовой оболочки и конформная $N=(4,4)$ супергравитация были описаны в $S U(2) \times S U(2)$ гармоническом суперпространстве в работах [17].
} 
но-временной четностью [20]-[22]). Как отмечено выше, основное различие между ними при описании в $N=(4,4)$ суперпространстве состоит в том, что группа внутренних автоморфизмов реализована на них по-разному [20], [21]. Одна из целей настояшей работы - показать, что сушествует свобода в определении преобразований дополнительной $N=(2,2)$ суперсимметрии на паре $N=(2,2)$ кирального суперполя и кирального суперполя с твистом. Именно она ответственна за сушествование нескольких неэквивалентных $N=(4,4)$ мультиплетов с твистом в рамках формулировки в двумерном $N=(2,2)$ суперпространстве.

В нашей предыдушей работе [21] было дано описание четырех типов $N=(4,4)$ мультиплетов с твистом в $S U(2) \times S U(2)$ гармоническом суперпространстве вне массовой оболочки и были построены обшие сигма-модельные суперполевые действия для них. В качестве примера был расмотрен специальный случай сигма-моделей ВЗНВ-типа на групповом многообразии $S U(2) \times U(1)$ для мультиплетов одного сорта. Было также показано, что обшее сигма-модельное действие для любой пары разных мультиплетов с твистом распадается в сумму сигма-модельных действий для отдельных мультиплетов.

Во многих случаях формулировки $N=(4,4)$ суперсимметричных моделей в $N=$ $(2,2)$ суперпространстве оказываются более наглядными, чем $N=(4,4)$ формулировки. В настояшей работе дано описание различных типов мультиплетов с твистом в двумерном $N=(2,2)$ суперпространстве $\mathbb{R}^{(1,1 \mid 2,2)}$. Подход $N=(2,2)$ суперпространства позволяет сушественно прояснить ряд утверждений и заключений, сделанных в работе [21].

Во втором разделе приведено описание $N=(4,4)$ мультиплетов с твистом в обычном $N=(4,4), 2 D$ суперпространстве $\mathbb{R}^{(1,1 \mid 4,4)}$. В разделе 3 условия неприводимости для этих мультиплетов сформулированы в $N=(2,2), 2 D$ суперпространстве $\mathbb{R}^{(1,1 \mid 2,2)}$. Показано, что во всех случаях эти условия приводят к тому, что данный $N=(4,4)$ мультиплет с твистом представляется парой $N=(2,2)$ кирального суперполя и кирального суперполя с твистом. На $N=(2,2)$ языке неэквивалентные $N=(4,4)$ мультиплеты с твистом отличаются друг от друга трансформационными свойствами этих пар относительно дополнительной $N=(2,2)$ суперсимметрии. Действия для мультиплетов с твистом строятся в виде интегралов по $N=(2,2)$ суперпространству от вещественных функций, зависяших от соответствуюших $N=(2,2)$ суперполей. Эти функции удовлетворяют определенным дифференциальным связям, возникаюшим как условия скрытой суперсимметрии. В четвертом разделе рассматриваются $N=(2,2)$ суперполевые действия двух типов. Лагранжевы плотности в этих действиях строятся либо из $N=(4,4)$ мультиплетов, принадлежаших самодуальной паре таких мультиплетов, либо из мультиплетов, относяшихся к несамодуальным парам ${ }^{2)}$. Мы показываем, что в обоих случаях сигма-модельные действия редуцируются к сумме действий для отдельных $N=(4,4)$ мультиплетов, и это свойство сохраняется при включении в действие большего числа неэквивалентных мультиплетов. Мы также показываем, что единственная возможность получить взаимодействие различных $N=(4,4)$ мультиплетов состо-

\footnotetext{
${ }^{2)}$ Определение "самодуальных" мультиплетов с твистом было дано в работах [14], [20], [21] (см. также раздел 2).
} 
ит в добавлении к сигма-модельному действию $N=(4,4)$ суперсимметричных потенциальных (или массовых) членов, которые строятся из мультиплетов, принадлежащих самодуальной паре. Мультиплеты, принадлежащие несамодуальным парам, не могут взаимодействовать друг с другом ни через потенциальные, ни через сигма-модельные члены. Проведенный анализ полностью подтверждает аналогичные результаты, полученные в рамках $N=(4,4)$ гармонического суперпространства [21]. Результаты работы суммированы в заключительном разделе 5 .

\section{2. МУЛЬТИПЛЕТЫ С ТВИСТОМ В $N=(4,4), 2 D$ СУПЕРПРОСТРАНСТВЕ}

Вначале, следуя работам [16], [21], напомним основные факты об $N=(4,4), 2 D$ суперсимметриии.

Стандартное вешественное $N=(4,4), 2 D$ суперпространство в параметризации светового конуса задается следуюшим набором координат:

$$
\mathbb{R}^{(1,1 \mid 4,4)}=\left(Z_{\mathrm{L}}, Z_{\mathrm{R}}\right)=\left(z^{++}, \theta^{+i \underline{k}}, z^{--}, \theta^{-a \underline{b}}\right) .
$$

Здесь “+”, “-”- индексы координат светового конуса, $i, \underline{k}, a, \underline{b}$ - дублетные индексы четырех коммутирующих групп $S U(2)$, составляющих полную группу автоморфизмов $S O(4)_{\mathrm{L}} \times S O(4)_{\mathrm{R}}$ двумерной $N=(4,4)$ супералгебры Пуанкаре. Спинорные ковариантные производные удовлетворяют антикоммутационным соотношениям

$$
\left\{D_{i \underline{k}}, D_{j \underline{l}}\right\}=2 i \varepsilon_{i j} \varepsilon_{\underline{k} \underline{l}} \partial_{++}, \quad\left\{D_{a \underline{b}}, D_{c \underline{d}}\right\}=2 i \varepsilon_{a c} \varepsilon_{\underline{b} \underline{d}} \partial_{--},
$$

где

$$
D_{i \underline{k}}=\frac{\partial}{\partial \theta^{i \underline{k}}}+i \theta_{i \underline{k}} \partial_{++}, \quad D_{a \underline{b}}=\frac{\partial}{\partial \theta^{a} \underline{b}}+i \theta_{a \underline{b}} \partial_{--} .
$$

Далее для спинорных производных и грассмановых координат будут использоваться как квартетные, так и дублетные обозначения. Для компактности записи индексы световых координат в квартетном представлении будем опускать. Эти два представления связаны между собой следуюшим образом:

$$
\begin{aligned}
& \left(\theta^{+i}, \bar{\theta}^{+i}\right) \equiv \theta^{i \underline{k}}, \quad\left(\theta^{i \underline{k}}\right)^{\dagger}=\varepsilon_{i l} \varepsilon_{\underline{k} \underline{n}} \theta^{l} \underline{\underline{n}}, \\
& \left(\theta^{-a}, \bar{\theta}^{-a}\right) \equiv \theta^{a} \underline{b}, \quad\left(\theta^{a} \underline{b}\right)^{\dagger}=\varepsilon_{a c} \varepsilon_{\underline{b}} \underline{d} \theta^{c} \underline{d}, \\
& \left(D_{+i}, \bar{D}_{+i}\right) \equiv D_{i \underline{k}}, \quad\left(D_{i \underline{k}}\right)^{\dagger}=-\varepsilon^{i l} \varepsilon^{\underline{k} \underline{n}} D_{l \underline{n}}, \\
& \left(D_{-a}, \bar{D}_{-a}\right) \equiv D_{a \underline{b}}, \quad\left(D_{a \underline{b}}\right)^{\dagger}=-\varepsilon^{a c} \varepsilon^{\underline{b} \underline{d}} D_{c \underline{d}} \text {. }
\end{aligned}
$$

Символ † в этих формулах обозначает комплексное сопряжение.

Суперсимметричные $N=(4,4)$ сигма-модели с кручением могут быть сформулированы либо в терминах $N=(2,2)$ суперполевых пар, состояших из кирального суперполя и кирального суперполя с твистом, либо в терминах $N=(4,4)$ суперполей, подчиненных определенным связям. Каждый из этих суперполевых наборов дает эквивалентное описание $N=(4,4)$ мультиплета с твистом вне массовой оболочки. 
В двумерном $N=(2,2)$ суперпространстве эти модели описываются действием, которое представляется в виде интеграла от вешественного потенциала [2], [7], [9], зависящего от нескольких копий кирального суперполя и кирального суперполя с твистом. Вещественный потенциал удовлетворяет набору дифференциальных связей, обеспечивающих инвариантность действия вне массовой оболочки относительно дополнительной $N=(2,2)$ суперсимметрии. Преобразования этой дополнительной $N=(2,2)$ суперсимметрии переводят киральные суперполя в киральные суперполя с твистом, и наоборот. Вместе с явной $N=(2,2)$ суперсимметрией они образуют полную $N=(4,4)$ суперсимметрию.

С другой стороны, $N=(4,4)$ супермультиплет с твистом в суперпространстве $\mathbb{R}^{(1,1 \mid 4,4)}$ описывается вешественным квартетным суперполем, подчиненным соответствуюшим условиям неприводимости [1], [2], [4], [23]. Эти условия оставляют $(8+8)$ независимых компонент в полном наборе полей вещественного квартетного суперполя, что как раз отвечает неприводимому составу $N=(4,4)$ мультиплета с твистом вне массовой оболочки.

Общие сигма-модельные действия с $N=(4,4)$ суперсимметрией, которые изучались ранее как в суперпространстве $\mathbb{R}^{(1,1 \mid 2,2)}[2]$, так и в суперпространстве $\mathbb{R}^{(1,1 \mid 4,4)}[16]$, включали зависимость лиш от одного типа $N=(4,4)$ мультиплетов с твистом. Однако в действительности сушествует несколько типов таких мультиплетов, компонентные поля которых различаются трансформационными свойствами по отношению к полной группе $R$-симметрии (группе автоморфизмов) $S O(4)_{\mathrm{L}} \times S O(4)_{\mathrm{R}}$ двумерной $N=(4,4)$ супералгебры Пуанкаре. Сушествование таких неэквивалентных мультиплетов было впервые замечено в работах [13], [19], [20].

Рассмотрим, следуя работам [19], [21], четыре типа мультиплетов с твистом в суперпространстве $\mathbb{R}^{(1,1 \mid 4,4)}[16]$ в соответствии с четырьмя возможностями объединения дублетных индексов $S U(2)$-факторов левой и правой подгрупп $S O(4)$ в полной группе автоморфизмов $S O(4)_{\mathrm{L}} \times S O(4)_{\mathrm{R}}$ :

$$
\hat{q}^{i a}, \quad \hat{q}^{i \underline{a}}, \quad \hat{q}^{\underline{i} a}, \quad \hat{q}^{\underline{i}} \underline{a} .
$$

Эти мультиплеты являются вешественными относительно комплексного сопряжения в суперпространстве $\mathbb{R}^{(1,1 \mid 4,4)}$ :

$$
\begin{array}{ll}
\left(\hat{q}^{i a}\right)^{\dagger}=\varepsilon_{i k} \varepsilon_{a b} \hat{q}^{k b}, & \left(\hat{q}^{i \underline{a}}\right)^{\dagger}=\varepsilon_{i k} \varepsilon_{a} \underline{b} \hat{q}^{k \underline{b}}, \\
\left(\hat{q}^{\underline{i} a}\right)^{\dagger}=\varepsilon_{\underline{i} \underline{k}} \varepsilon_{a b} \hat{q}^{\underline{k} b}, & \left(\hat{q}^{\underline{i} \underline{a}}\right)^{\dagger}=\varepsilon_{\underline{i} \underline{k}} \varepsilon_{\underline{a}} \underline{b} \hat{q}^{\underline{k}} \underline{b} .
\end{array}
$$

Условия неприводимости, оставляюшие $(8+8)$ независимых компонент в каждом из суперполей, имеют вид

$$
\begin{array}{ll}
D^{\left(k \underline{k} \hat{q}^{i) a}=0,\right.} & D^{(b \underline{b}} \hat{q}^{i a)}=0, \\
D^{(k \underline{k}} \hat{q}^{i) \underline{a}}=0, & D^{b(\underline{b}} \hat{q}^{i \underline{a})}=0, \\
D^{k(\underline{k}} \hat{q}^{\underline{i}) a}=0, & D^{(b \underline{b}} \hat{q}^{\underline{i} a)}=0, \\
D^{k(\underline{k}} \hat{q}^{\underline{i})} \underline{a}=0, & D^{b(\underline{b}} \hat{q}^{\underline{i} \underline{a})}=0 .
\end{array}
$$


Символ ( ) в этих формулах обозначает симметризацию по соответствующим индексам.

Для описания этих мультиплетов наиболее подходяшим является $N=(4,4), S U(2) \times$ $S U(2)$ гармоническое суперпространство, содержашее два набора независимых гармонических переменных [16], [21]. Для каждого типа $N=(4,4)$ мультиплета с твистом обшее сигма-модельное действие вне массовой оболочки записывается как интеграл по аналитическому бигармоническому суперпространству [16], [21],которое образует подпространство в общем $S U(2) \times S U(2)$ гармоническом суперпространстве.

В работе [21] было показано, что в аналитическом суперпространстве существует возможность обобщения сигма-модельных действий, зависящих от одного типа мультиплетов с твистом, на случай зависимости от двух и большего числа мультиплетов разного типа. Основной целью данной работы является формулировка этих результатов на более прозрачном $N=(2,2)$ суперполевом языке.

В заключение этого раздела напомним определение самодуальной пары мультиплетов с твистом из набора (2.5). Пара мультиплетов назьвается самодуальной, если входящие в нее суперполя не имеют общих $S U(2)$ дублетных индексов. Так, $\left(\hat{q}^{i a}, \hat{q}^{\underline{i}} \underline{a}\right)$ и $\left(\hat{q}^{i \underline{a}}, \hat{q}^{\underline{\underline{i}} a}\right)$ - самодуальные пары. Их отличительная особенность состоит в том, что физические бозонные поля одного из суперполей, входяших в пару, имеют тот же самый $S U(2)$ состав, что и вспомогательные поля другого суперполя из той же пары. Все остальные пары мультиплетов будем называть несамодуальными.

\section{3. МУЛЬТИПЛЕТЫ С ТВИСТОМ В $N=(2,2), 2 D$ СУПЕРПРОСТРАНСТВЕ}

В этом разделе будет дано описание разных типов $N=(4,4)$ мультиплетов с твистом в двумерном $N=(2,2)$ суперпространстве и показано, в чем состоит различие между ними. Каждый из таких мультиплетов задается парой $N=(2,2)$ кирального суперполя и кирального суперполя с твистом, которые, однако, имеют разные трансформационные свойства относительно преобразований дополнительной $N=(2,2)$ суперсимметрии. Затем будут построены обшие $N=(4,4)$ суперсимметричные сигма-модельные действия для каждого типа мультиплетов с твистом. Эти действия записываются в виде интегралов по $N=(2,2)$ суперпространству от действительных функций, зависяших от $N=(2,2)$ кирального суперполя и кирального суперполя с твистом. Эти суперполевые лагранжевы плотности подчинены определенным дифференциальными связям.

3.1. Преобразования суперсимметрии и связи. Перейдем к эквивалентной записи для $\mathbb{R}^{(1,1 \mid 4,4)}$ грассмановых координат и спинорных производных (2.1), (2.4):

$$
\begin{aligned}
& \theta^{+i}=\left(\theta^{+}, \eta^{+}\right), \quad \bar{\theta}_{i}^{+}=\left(\bar{\theta}^{+}, \bar{\eta}^{+}\right), \quad \theta^{-a}=\left(\theta^{-}, \xi^{-}\right), \quad \bar{\theta}_{a}^{-}=\left(\bar{\theta}^{-}, \bar{\xi}^{-}\right), \\
& D_{+i}=\left(D_{+}, d_{+}\right), \bar{D}_{+}^{i}=\left(\bar{D}_{+}, \bar{d}_{+}\right), D_{-a}=\left(D_{-}, d_{-}\right), \bar{D}_{-}^{a}=\left(\bar{D}_{-}, \bar{d}_{-}\right) \text {. }
\end{aligned}
$$

Координаты $\theta$ и спинорные производные $D$ соответствуют явной $N=(2,2)$ суперсимметрии, а координаты $\eta, \xi$ и спинорные производные $d$ - скрытой $N=(2,2)$ суперсимметрии (соотношения, связьваюшие $N=(4,4)$ ковариантные производные в квартетных обозначениях $(2.4)$ с их $N=(2,2)$ аналогами, приведены в приложении А). 
Вешественное $N=(2,2), 2 D$ суперпространство $\mathbb{R}^{(1,1 \mid 2,2)}$ параметризуется следующим набором координат:

$$
\mathbb{R}^{(1,1 \mid 2,2)}=\left(z^{++}, \theta^{+}, \bar{\theta}^{+}, z^{--}, \theta^{-}, \bar{\theta}^{-}\right) .
$$

Перепишем условия неприводимости для $N=(4,4)$ мультиплетов с твистом $(2.7)$ $(2.10)$ в суперпространстве $\mathbb{R}^{(1,1 \mid 2,2)}$. Для этого с учетом условий вешественности $(2.6)$ введем комплексные $N=(4,4)$ суперполя

$$
\begin{aligned}
& \hat{q}^{11}=\mathbf{A}, \quad \hat{q}^{12}=\mathbf{B}, \quad \hat{q}^{21}=-\overline{\mathbf{B}}, \quad \hat{q}^{22}=\overline{\mathbf{A}}, \\
& \hat{q}^{1} \underline{1}=\mathbf{a}, \quad \hat{q}^{1} \underline{2}=\mathbf{b}, \quad \hat{q}^{2} \underline{1}=-\overline{\mathbf{b}}, \quad \hat{q}^{2} \underline{2}=\overline{\mathbf{a}}, \\
& \hat{q}^{11}=\mathcal{A}, \quad \hat{q}^{12}=\mathcal{B}, \quad \hat{q}^{\underline{2}}{ }^{1}=-\overline{\mathcal{B}}, \quad \hat{q}^{\underline{2}}{ }^{2}=\overline{\mathcal{A}}, \\
& \hat{q}^{1} \underline{1}=\mathrm{A}, \quad \hat{q}^{\underline{1}} \underline{\underline{2}}=\mathrm{B}, \quad \hat{q}^{\underline{2}} \underline{1}=-\overline{\mathrm{B}}, \quad \hat{q}^{\underline{2}} \underline{\underline{2}}=\overline{\mathrm{A}} .
\end{aligned}
$$

После разложения этих суперполей по дополнительным грассмановым координатам $\eta^{ \pm}$, $\xi^{ \pm}$(и комплексно-сопряженным координатам) и использования связей (2.7)-(2.10) замечаем, что только первые компоненты в каждом из $N=(4,4)$ суперполей являются независимыми $N=(2,2)$ суперполями. Все остальные $N=(2,2)$ суперполевые компоненты в разложениях по дополнительным грассмановым координатам выражаются через спинорные производные $D$ соответствуюших первых компонент. Низшие компоненты, как следует из связей $(2.7)-(2.10)$, являются киральными $N=(2,2)$ суперполями и киральными $N=(2,2)$ суперполями с твистом.

Так, для мультиплета $\hat{q}^{i a}$ имеем (для остальных мультиплетов аналогичные условия приведены в приложении $\mathrm{A}$ )

$$
\begin{array}{llll}
\bar{D}_{+} A=0, & \bar{D}_{-} A=0, & \bar{D}_{+} B=0, & D_{-} B=0, \\
D_{+} \bar{A}=0, & D_{-} \bar{A}=0, & D_{+} \bar{B}=0, & \bar{D}_{-} \bar{B}=0,
\end{array}
$$

где

$$
A=\left.\mathbf{A}\right|_{\eta=\xi=0}, \quad \bar{A}=\left.\overline{\mathbf{A}}\right|_{\eta=\xi=0}, \quad B=\left.\mathbf{B}\right|_{\eta=\xi=0}, \quad \bar{B}=\left.\overline{\mathbf{B}}\right|_{\eta=\xi=0} .
$$

Чтобы увидеть различия между неэквивалентными $N=(4,4)$ мультиплетами с твистом в суперпространстве $\mathbb{R}^{(1,1 \mid 2,2)}$, приведем явный вид разложения по дополнительным грассмановым координатам тех $N=(4,4)$ суперполей, чьи первые компоненты являются киральными $N=(2,2)$ суперполями:

$$
\begin{aligned}
\mathbf{A} & =A-\bar{\eta}^{+} \bar{D}_{+} \bar{B}+\bar{\xi}^{-} \bar{D}_{-} B+\cdots, & & \mathbf{A}=\mathrm{a}+\eta^{+} \bar{D}_{+} \overline{\mathrm{b}}-\xi^{-} \bar{D}_{-} \mathrm{b}+\cdots, \\
\mathbf{a} & =a-\bar{\eta}^{+} \bar{D}_{+} \bar{b}-\xi^{-} \bar{D}_{-} b+\cdots, & & \mathcal{A}=\alpha+\eta^{+} \bar{D}_{+} \bar{\beta}+\bar{\xi}^{-} \bar{D}_{-} \beta+\cdots,
\end{aligned}
$$

где

$$
A=\left.\mathbf{A}\right|_{\eta=\xi=0}, \quad a=\left.\mathbf{a}\right|_{\eta=\xi=0}, \quad \alpha=\left.\mathcal{A}\right|_{\eta=\xi=0}, \quad a=\left.\mathrm{A}\right|_{\eta=\xi=0} .
$$

В суперпространстве $\mathbb{R}^{(1,1 \mid 4,4)}$ преобразования суперсимметрии $N=(4,4)$ суперполя $\Phi$ выглядят следуюшим образом (явньй вид сответствуюших генераторов $Q$ приведен в приложении A):

$$
\delta \Phi=i\left(\varepsilon^{+k} Q_{+k}-\bar{\varepsilon}_{k}^{+} \bar{Q}_{+}^{k}+\varepsilon^{-a} Q_{-a}-\bar{\varepsilon}_{a}^{-} \bar{Q}_{-}^{a}\right) \Phi .
$$


При редукции суперпространства $\mathbb{R}^{(1,1 \mid 4,4)}$ к подпространству $\mathbb{R}^{(1,1 \mid 2,2)}$ половина суперсимметрий становится неявной, и их преобразования включают спинорные производные $D$. Эти дополнительные суперсимметрии смешивают между собой независимые $N=(2,2)$ суперполевые компоненты $N=(4,4)$ суперполей, определенные формулами (3.9), (А.10)-(А.12).

Подставляя разложение по дополнительным грассмановым переменным для каждого $N=(4,4)$ суперполя в закон преобразования $(3.11)$ и выбирая значения индексов $k=$ $a=2$ (которые отвечают генераторам и инфинитезимальным параметрам скрытой $N=$ $(2,2)$ суперсимметрии), находим законы преобразований независимых компонент этих суперполей относительно дополнительной $N=(2,2)$ суперсимметрии:

$$
\begin{aligned}
\delta A=\bar{\varepsilon}^{+} \bar{D}_{+} \bar{B}-\bar{\varepsilon}^{-} \bar{D}_{-} B, & \delta \mathrm{a}=-\varepsilon^{+} \bar{D}_{+} \overline{\mathrm{b}}+\varepsilon^{-} \bar{D}_{-} \mathrm{b}, \\
\delta \bar{A}=-\varepsilon^{+} D_{+} B+\varepsilon^{-} D_{-} \bar{B}, & \delta \overline{\mathrm{a}}=\bar{\varepsilon}^{+} D_{+} \mathrm{b}-\bar{\varepsilon}^{-} D_{-} \overline{\mathrm{b}}, \\
\delta B=-\bar{\varepsilon}^{+} \bar{D}_{+} \bar{A}-\varepsilon^{-} D_{-} A, & \delta \mathrm{b}=\varepsilon^{+} \bar{D}_{+} \overline{\mathrm{a}}+\bar{\varepsilon}^{-} D_{-} \mathrm{a}, \\
\delta \bar{B}=\varepsilon^{+} D_{+} A+\bar{\varepsilon}^{-} \bar{D}_{-} \bar{A}, & \delta \overline{\mathrm{b}}=-\bar{\varepsilon}^{+} D_{+} \mathrm{a}-\varepsilon^{-} \bar{D}_{-} \overline{\mathrm{a}}, \\
\delta a=\bar{\varepsilon}^{+} \bar{D}_{+} \bar{b}+\varepsilon^{-} \bar{D}_{-} b, & \delta \alpha=-\varepsilon^{+} \bar{D}_{+} \bar{\beta}-\bar{\varepsilon}^{-} \bar{D}_{-} \beta, \\
\delta \bar{a}=-\varepsilon^{+} D_{+} b-\bar{\varepsilon}^{-} D_{-} \bar{b}, & \delta \bar{\alpha}=\bar{\varepsilon}^{+} D_{+} \beta+\varepsilon^{-} D_{-} \bar{\beta}, \\
\delta b=-\bar{\varepsilon}^{+} \bar{D}_{+} \bar{a}+\bar{\varepsilon}^{-} D_{-} a, & \delta \beta=\varepsilon^{+} \bar{D}_{+} \bar{\alpha}-\varepsilon^{-} D_{-} \alpha, \\
\delta \bar{b}=\varepsilon^{+} D_{+} a-\varepsilon^{-} \bar{D}_{-} \bar{a}, & \delta \bar{\beta}=-\bar{\varepsilon}^{+} D_{+} \alpha+\bar{\varepsilon}^{-} \bar{D}_{-} \bar{\alpha} .
\end{aligned}
$$

Здесь выписаны законы преобразований независимых $N=(2,2)$ суперполей, представляющих $N=(4,4)$ мультиплеты из самодуальных пар $\left(\hat{q}^{i a}, \hat{q} \underline{\underline{i}} \underline{a}\right)$ и $\left(\hat{q}^{i \underline{a}}, \hat{q}^{\underline{i}} a\right)$, соответственно.

Из $(3.12),(3.13)$ следует, что разные типы $N=(4,4)$ мультиплетов с твистом в суперпространстве $\mathbb{R}^{(1,1 \mid 2,2)}$ отличаются друг от друга законами преобразования соответствующих киральных суперполей и киральных суперполей с твистом по отношению к дополнительной $N=(2,2)$ суперсимметрии. Эти законы преобразования специфичны для каждого мультиплета.

Таким образом, каждый тип $N=(4,4)$ мультиплетов с твистом в суперпространстве $\mathbb{R}^{(1,1 \mid 2,2)}$ описывается парой суперполей: кирального $N=(2,2)$ суперполя и кирального $N=(2,2)$ суперполя с твистом. В то же время дополнительная $N=(2,2)$ суперсимметрия реализована по-разному на $N=(2,2)$ суперполях из неэквивалентных $N=(4,4)$ мультиплетов. Более того, для $N=(2,2)$ суперполей одного сорта (киральных или киральных с твистом), но принадлежаших разным $N=(4,4)$ мультиплетам, никакими переопределениями параметров преобразований и /или суперполей нельзя одновременно привести преобразования неявной $N=(2,2)$ суперсимметрии к одному и тому же виду.

Таким образом, основная отличительная черта неэквивалентных мультиплетов с твистом в $N=(2,2)$ суперполевом описании заключается в различии реализаций дополнительной неявной $N=(2,2)$ суперсимметрии. 
Возврашаясь к полной группе автоморфизмов $S O(4)_{\mathrm{L}} \times S O(4)_{\mathrm{R}}$ двумерной $N=(4,4)$ супералгебры Пуанкаре, следует отметить, что она имеет различные реализации на неэквивалентных $N=(4,4)$ мультиплетах с твистом как в $N=(2,2), 2 D$, так и $N=$ $(4,4), 2 D$ суперпространствах. При описании в суперпространстве $\mathbb{R}^{(1,1 \mid 2,2)}$ непосредственно наблюдаема только симметрия относительно подгруппы $U(1)_{\mathrm{L}} \times U(1)_{\mathrm{R}}$ полной группы автоморфизмов $S O(4)_{\mathrm{L}} \times S O(4)_{\mathrm{R}}$.

3.2. Действие для мультиплета $\hat{q}^{i a}$. Обшее действие для $k$ киральных суперполей $C^{k}$ и $n$ киральных суперполей с твистом $T^{n}$ записывается в суперпространстве $\mathbb{R}^{(1,1 \mid 2,2)}$ как интеграл от некоторой вещественной функции $K$ :

$$
S_{(2,2)}=\int \mu K\left(C^{k}, \bar{C}^{k}, T^{n}, \bar{T}^{n}\right),
$$

где

$$
\mu=d^{2} x d^{2} \theta^{+} d^{2} \theta^{-}=d^{2} x d \theta^{+} d \bar{\theta}^{+} d \theta^{-} d \bar{\theta}^{-}
$$

- мера интегрирования. Суперпотенциал $K$ инвариантен относительно обобщенного кэлерова преобразования:

$$
\delta K=f\left(C^{k}, T^{n}\right)+g\left(C^{k}, \bar{T}^{n}\right)+\bar{f}\left(\bar{C}^{k}, \bar{T}^{n}\right)+\bar{g}\left(\bar{C}^{k}, T^{n}\right) .
$$

Если это действие описывает теорию, обладающую только $N=(2,2)$ суперсимметрией, то число киральных суперполей и киральных суперполей с твистом может быть произвольным, $k \neq n$. К действию (3.14) без введения центральных зарядов можно добавить скалярный потенциал, который включает в себя две голоморфные функции $P_{1}\left(C^{k}\right)$, $P_{2}\left(T^{n}\right)$

$S_{(2,2)}^{\mathrm{pot}}=i m \int \mu\left\{\left(\bar{\theta}^{+} \bar{\theta}^{-}\right) P_{1}\left(C^{k}\right)+\left(\theta^{+} \theta^{-}\right) \bar{P}_{1}\left(\bar{C}^{k}\right)+\left(\bar{\theta}^{+} \theta^{-}\right) P_{2}\left(T^{n}\right)+\left(\theta^{+} \bar{\theta}^{-}\right) \bar{P}_{2}\left(\bar{T}^{n}\right)\right\}$.

Несмотря на присутствие грассмановых координат $\theta$, скалярный потенциал (3.17) инвариантен относительно преобразований явной $N=(2,2)$ суперсимметрии как следствие того, что входящие в него суперполя являются киральными и киральными с твистом, т.е. удовлетворяют известным связям. После исключения вспомогательных полей из суммы двух действий (3.14) и (3.17) в компонентном действии возникают скалярные потенциальные члены физических бозонных полей.

Предположим, что киральные суперполя $C$ (или антикиральные $\bar{C}$ ) и киральные суперполя с твистом $T$ (или антикиральные с твистом $\bar{T}$ ) объединяются в один и тот же фиксированный тип $N=(4,4)$ мультиплета с твистом. Тогда инвариантность действия (3.14) относительно дополнительной $N=(2,2)$ суперсимметрии требует, во-первых, равенства числа киральных суперполей и киральных суперполей с твистом, $k=n$, и, во-вторых, приводит к дифференциальным связям для функции $K$. Эти связи вьглядят следующим образом [2]:

$$
\begin{aligned}
\frac{\partial^{2} K}{\partial C^{l} \partial \bar{C}^{k}}-\frac{\partial^{2} K}{\partial C^{k} \partial \bar{C}^{l}} & =0, \quad \frac{\partial^{2} K}{\partial T^{l} \partial \bar{T}^{k}}-\frac{\partial^{2} K}{\partial T^{k} \partial \bar{T}^{l}}=0 \\
\frac{\partial^{2} K}{\partial C^{(k} \partial \bar{C}^{l)}}+\frac{\partial^{2} K}{\partial T^{(l} \partial \bar{T}^{k)}} & =0 .
\end{aligned}
$$


В частном случае, когда потенциал $K$ зависит только от одной пары суперполей - кирального суперполя и кирального суперполя с твистом $(m=1)$ - связи (3.18), (3.19) сводятся к единственному четырехмерному уравнению Лапласа

$$
\frac{\partial^{2} K}{\partial C \partial \bar{C}}+\frac{\partial^{2} K}{\partial T \partial \bar{T}}=0
$$

Таким образом, для вешественного потенциала $K$, удовлетворяюшего условиям (3.18), (3.19) и включаюшего равное число киральных и киральных с твистом суперполей, действие

$$
S_{(4,4)}=\int \mu K\left(C^{m}, \bar{C}^{m}, T^{m}, \bar{T}^{m}\right)
$$

инвариантно относительно полной $N=(4,4)$ суперсимметрии.

Можно показать, что инвариантность потенциальных (массовых) членов в действии $(3.17)$ относительно $N=(4,4)$ суперсимметрии требует линейной зависимости функций $P_{1}, P_{2}$ от соответствующих суперполей:

$$
S_{(4,4)}^{m}=i m \int \mu\left\{\left(\bar{\theta}^{+} \bar{\theta}^{-}\right) C+\left(\theta^{+} \theta^{-}\right) \bar{C}+\left(\bar{\theta}^{+} \theta^{-}\right) T+\left(\theta^{+} \bar{\theta}^{-}\right) \bar{T}\right\} .
$$

Хотя в компонентном действии (3.22) присутствуют только члены, линейные по вспомогательным полям, после исключения последних в полном действии, содержащем также сигма-модельную часть, возникает скалярньй потенциал для физических бозонных полей (в случае нетривиальной метрики на пространстве физических бозонов) [1], [4], $[13],[16]$.

Проведенный анализ можно распространить на любой другой тип $N=(4,4)$ мультиплета с твистом. Если действие зависит от пар $N=(2,2)$ киральных и киральных с твистом суперполей, образующих один и тот же тип $N=(4,4)$ мультиплета с твистом, то дифференциальные условия для соответствующего потенциала $K$, вытекающие из требования дополнительной $N=(2,2)$ суперсимметрии, имеют такой же вид, как и (3.18), (3.19), независимо от типа мультиплета. Структура потенциальных членов вне массовой оболочки в этих случаях также однозначно фиксируется требованием инвариантности относительно дополнительной $N=(2,2)$ суперсимметрии. Они представляются суммой действий типа (3.22).

\section{4. ДЕЙСТВИЕ ДЛЯ ПАРЫ МУЛЬТИПЛЕТОВ С ТВИСТОМ}

4.1. Введение: свободное действие. В предыдущем разделе было показано, что киральные суперполя и киральные суперполя с твистом, образующие $N=(4,4)$ мультиплет с твистом, могут иметь разные трансформационные свойства относительно преобразований скрытой $N=(2,2)$ суперсимметрии. Как результат, соответствуюшие $N=(4,4)$ мультиплеты не эквивалентны друг другу.

Рассмотрим в суперпространстве $\mathbb{R}^{(1,1 \mid 2,2)}$ сигма-модельные действия для двух случаев, когда суперпотенциал $K$ содержит зависимость только от самодуальной или только от несамодуальной пары $N=(4,4)$ мультиплетов с твистом. Как будет показано ниже, в обоих вариантах соответствуюшие сигма-модельные действия сводятся к сумме 
сигма-модельных действий для отдельных мультиплетов с твистом. Представленные в этом разделе результаты полностью согласуются с полученными в работе [21] в подходе бигармонического суперпространства.

Преж де чем перейти к обшему рассмотрению, напомним, как в бигармоническом подходе выглядят действия, билинейные по $N=(4,4)$ суперполям с твистом [21]. Следует различать два случая. В первом случае общие квадратичные действия зависят только от одного типа мультиплета с твистом и априори содержат произвольные гармонические константы. Требование, чтобы эти действия были $N=(4,4)$ суперсимметричными, приводит к условиям для гармонических констант, и в результате соответствующие действия сводятся к свободным. Во втором случае билинейные действия зависят от разных типов $N=(4,4)$ мультиплетов с твистом. Изучение таких действий в работе [21] привело к заключению, что требование инвариантности относительно $N=(4,4)$ суперсимметрии сводит их к нулю.

Эти результаты могут быть воспроизведены в суперпространстве $\mathbb{R}^{(1,1 \mid 2,2)}$ в терминах киральных суперполей и киральных суперполей с твистом. Так, билинейное сигма-модельное действие, зависящее от кирального и кирального с твистом суперполей, которые принадлежат одному типу $N=(4,4)$ мультиплета с твистом,

$$
S_{(4,4)}^{\text {free }}=\int \mu\left(C^{m} \bar{C}^{m}-T^{m} \bar{T}^{m}\right),
$$

эквивалентно свободному $N=(4,4)$ суперсимметричному действию соответствующего мультиплета с твистом. В то же время действия, билинейные по таким парам киральных суперполей и киральных суперполей с твистом, которые относятся к разным типам $N=(4,4)$ мультиплетов, оказываются равными нулю в силу требования дополнительной $N=(2,2)$ суперсимметрии. Заметим, что относительный знак между двумя членами в (4.1) фиксируется этой дополнительной $N=(2,2)$ суперсимметрией единственным образом (компонентный лагранжиан, несмотря на знак минус в (4.1), положительно определен).

Ниже мы повторим в суперпространстве $\mathbb{R}^{(1,1 \mid 2,2)}$ обший анализ сигма-модельных действий для обоих случаев, т.е. как для несамодуальной, так и для самодуальной пары мультиплетов с твистом [21]. Единственность формы свободного действия (4.1) и несушествование $N=(4,4)$ суперсимметричных билинейных действий для $N=(2,2)$ суперполевых пар, представляющих разные $N=(4,4)$ мультиплеты с твистом, следуют из этого общего анализа.

4.2. Действие для несамодуальных мультиплетов с твистом. Рассмотрим действие для мультиплетов, принадлежащих разным самодуальным парам, $\hat{q}^{i a}$ и $\hat{q}^{i \underline{a}}$. Оно записывается как интеграл от вешественной функции $K$ по суперпространству $\mathbb{R}^{(1,1 \mid 2,2) \text { : }}$

$$
S_{(4,4)}=\int \mu K(A, \bar{A}, B, \bar{B}, a, \bar{a}, b, \bar{b}) .
$$

Действие (4.2) инвариантно относительно явной $N=(2,2)$ суперсимметрии, поскольку функция $K$ зависит только от $N=(2,2)$ суперполей. Оно также инвариантно относи- 
тельно обобшенных калибровочных кэлеровых преобразований:

$$
\delta K=f(A, B, a, b)+g(A, \bar{B}, a, \bar{b})+\bar{f}(\bar{A}, \bar{B}, \bar{a}, \bar{b})+\bar{g}(\bar{A}, B, \bar{a}, b) .
$$

Используя тот факт, что входящие в правую часть (4.3) суперполя являются киральными и киральными с твистом, а также определение меры интегрирования на суперпространстве $\mathbb{R}^{(1,1 \mid 2,2)}$, легко показать, что калибровочные функции в (4.3) не дают вклада в $N=(2,2)$ суперполевое действие. Требование, чтобы это действие было инвариантным относительно дополнительной $N=(2,2)$ суперсимметрии, которая реализуется на суперполях преобразованиями (3.12), (3.13), оказывается равносильным дополнительным условиям для $K$.

Общее условие инвариантности действия (4.2) относительно преобразований скрытой суперсимметрии может быть записано в виде

$$
\begin{aligned}
\delta S_{(4,4)}= & \int \mu\left\{\varepsilon^{+} D_{+} F-\bar{\varepsilon}^{+} \bar{D}_{+} \bar{F}+\varepsilon^{+} \bar{D}_{+} G-\bar{\varepsilon}^{+} D_{+} \bar{G}+\right. \\
& \left.+\varepsilon^{-} D_{-} H-\bar{\varepsilon}^{-} \bar{D}_{-} \bar{H}+\varepsilon^{-} \bar{D}_{-} P-\bar{\varepsilon}^{-} D_{-} \bar{P}\right\},
\end{aligned}
$$

причем функции от $N=(2,2)$ суперполей в правой части изначально являются произвольными. Вычисляя явно вариацию $\delta S_{(4,4)}$ относительно скрытой суперсимметрии и сравнивая коэффициенты при независимых инфинитезимальных параметрах в обеих частях равенства (4.4), получаем

$$
\begin{aligned}
& \varepsilon^{+} \Rightarrow \frac{\partial K}{\partial \bar{B}}=\frac{\partial F}{\partial A}, \frac{\partial K}{\partial \bar{A}}=-\frac{\partial F}{\partial B}, \frac{\partial K}{\partial \bar{b}}=\frac{\partial F}{\partial a}, \frac{\partial K}{\partial \bar{a}}=-\frac{\partial F}{\partial b}, \\
& \bar{\varepsilon}^{+} \Rightarrow \frac{\partial K}{\partial B}=\frac{\partial \bar{F}}{\partial \bar{A}}, \frac{\partial K}{\partial A}=-\frac{\partial \bar{F}}{\partial \bar{B}}, \frac{\partial K}{\partial b}=\frac{\partial \bar{F}}{\partial \bar{a}}, \frac{\partial K}{\partial a}=-\frac{\partial \bar{F}}{\partial \bar{b}}, \\
& \varepsilon^{-} \Rightarrow \frac{\partial K}{\partial B}=-\frac{\partial H}{\partial A}, \frac{\partial K}{\partial \bar{A}}=\frac{\partial H}{\partial \bar{B}}, \frac{\partial K}{\partial \bar{b}}=-\frac{\partial P}{\partial \bar{a}}, \frac{\partial K}{\partial a}=\frac{\partial P}{\partial b}, \\
& \bar{\varepsilon}^{-} \Rightarrow \frac{\partial K}{\partial \bar{B}}=-\frac{\partial \bar{H}}{\partial \bar{A}}, \frac{\partial K}{\partial A}=\frac{\partial \bar{H}}{\partial B}, \frac{\partial K}{\partial b}=-\frac{\partial \bar{P}}{\partial a}, \frac{\partial K}{\partial \bar{a}}=\frac{\partial \bar{P}}{\partial \bar{b}} .
\end{aligned}
$$

Кроме этих связей, функции в правой части (4.4) подчиняются дополнительным условиям аналитичности:

$$
\begin{aligned}
& \varepsilon^{+} \Rightarrow G=0, \quad \bar{\varepsilon}^{+} \Rightarrow \bar{G}=0, \\
& \varepsilon^{-} \Rightarrow \frac{\partial H}{\partial a}=0, \quad \frac{\partial H}{\partial \bar{b}}=0, \quad \frac{\partial P}{\partial \bar{A}}=0, \quad \frac{\partial P}{\partial B}=0, \\
& \bar{\varepsilon}^{-} \Rightarrow \frac{\partial \bar{H}}{\partial \bar{a}}=0, \quad \frac{\partial \bar{H}}{\partial b}=0, \quad \frac{\partial \bar{P}}{\partial A}=0, \quad \frac{\partial \bar{P}}{\partial \bar{B}}=0 .
\end{aligned}
$$

Условия интегрируемости для потенциала $K$, следующие из уравнений $(4.5)$, имеют вид

$$
\begin{array}{rlrl}
\frac{\partial^{2} K}{\partial A \partial \bar{A}}+\frac{\partial^{2} K}{\partial B \partial \bar{B}}=0, & & \frac{\partial^{2} K}{\partial a \partial \bar{a}}+\frac{\partial^{2} K}{\partial b \partial \bar{b}}=0, \\
\frac{\partial^{2} K}{\partial a \partial \bar{B}}-\frac{\partial^{2} K}{\partial \bar{b} \partial A}=0, & \frac{\partial^{2} K}{\partial \bar{a} \partial B}-\frac{\partial^{2} K}{\partial b \partial \bar{A}}=0, \\
\frac{\partial^{2} K}{\partial a \partial \bar{A}}+\frac{\partial^{2} K}{\partial \bar{b} \partial B}=0, & \frac{\partial^{2} K}{\partial \bar{a} \partial A}+\frac{\partial^{2} K}{\partial b \partial \bar{B}}=0 .
\end{array}
$$


Аналогично из уравнений (4.6), (4.8) находим дальнейшие ограничения для $K$ :

$$
\begin{array}{llll}
\frac{\partial^{2} K}{\partial a \partial B}=0, & \frac{\partial^{2} K}{\partial \bar{b} \partial B}=0, & \frac{\partial^{2} K}{\partial a \partial \bar{A}}=0, & \frac{\partial^{2} K}{\partial \bar{b} \partial \bar{A}}=0, \\
\frac{\partial^{2} K}{\partial \bar{a} \partial \bar{B}}=0, & \frac{\partial^{2} K}{\partial b \partial \bar{B}}=0, & \frac{\partial^{2} K}{\partial \bar{a} \partial A}=0, & \frac{\partial^{2} K}{\partial b \partial A}=0,
\end{array}
$$

и, кроме них, такие же два уравнения Лапласа, как в первой строке в (4.9).

Для того чтобы найти решение условий интегрируемости для $K$, удобно ввести следуюшие дублеты $N=(2,2)$ суперполей:

$$
a^{\alpha}=(a, \bar{b}), \quad A^{\alpha}=(\bar{A}, B), \quad \bar{a}^{\alpha}=(\bar{a}, b), \quad \bar{A}^{\alpha}=(A, \bar{B}),
$$

где $\alpha, \beta=1,2$. В этих новых переменных система уравнений (4.9), (4.10) становится более компактной:

$$
\begin{aligned}
\frac{\partial^{2} K}{\partial A \partial \bar{A}}+\frac{\partial^{2} K}{\partial B \partial \bar{B}} & =0, & \frac{\partial^{2} K}{\partial a \partial \bar{a}}+\frac{\partial^{2} K}{\partial b \partial \bar{b}} & =0, \\
\frac{\partial^{2} K}{\partial a^{\alpha} \partial A^{\beta}} & =0, & \frac{\partial^{2} K}{\partial \bar{a}^{\alpha} \partial \bar{A}^{\beta}} & =0, \\
\varepsilon^{\alpha \beta} \frac{\partial^{2} K}{\partial a^{\alpha} \partial \bar{A}^{\beta}} & =0, & \varepsilon^{\alpha \beta} \frac{\partial^{2} K}{\partial \bar{a}^{\alpha} \partial A^{\beta}} & =0 .
\end{aligned}
$$

Решение уравнений (4.13), (4.14) имеет вид (детали приведены в приложении Б)

$$
K\left(A^{\alpha}, \bar{A}^{\alpha}, a^{\alpha}, \bar{a}^{\alpha}\right)=T\left(A^{\alpha}, \bar{A}^{\alpha}\right)+h\left(a^{\alpha}, \bar{a}^{\alpha}\right) .
$$

Дополнительно каждый член в правой части (4.15) подчиняется своему четырехмерному уравнению Лапласа и, таким образом, связи (4.12) также удовлетворяются.

4.3. Действие для самодуальных мультиплетов с твистом. Для самодуальной пары мультиплетов с твистом $\hat{q}^{\underline{i} a}$ и $\hat{q}^{i \underline{a}}$ общее $N=(2,2)$ суперсимметричное действие записывается в суперпространстве $\mathbb{R}^{(1,1 \mid 2,2)}$ в виде

$$
S_{(4,4)}=\int \mu K(a, \bar{a}, b, \bar{b}, \alpha, \bar{\alpha}, \beta, \bar{\beta}) .
$$

Действие (4.16) инвариантно относительно обобщенных кэлеровых преобразований:

$$
\delta K=f(a, b, \alpha, \beta)+g(a, \bar{b}, \alpha, \bar{\beta})+\bar{f}(\bar{a}, \bar{b}, \bar{\alpha}, \bar{\beta})+\bar{g}(\bar{a}, b, \bar{\alpha}, \beta) .
$$

Как и в случае несамодуальных мультиплетов, требование, чтобы действие обладало инвариантностью относительно дополнительной $N=(2,2)$ суперсимметрии, приводит 
к дифференциальным условиям для потенциала $K$. Чтобы их найти, воспользуемся обшим условием инвариантности (4.4) (обозначая функции в правой части теми же буквами) и законами преобразований суперполей (3.13). В результате получим

$$
\begin{aligned}
& \varepsilon^{+} \Rightarrow \frac{\partial K}{\partial \bar{b}}=\frac{\partial F}{\partial a}, \quad \frac{\partial K}{\partial \bar{a}}=-\frac{\partial F}{\partial b}, \quad \frac{\partial K}{\partial \beta}=\frac{\partial G}{\partial \bar{\alpha}}, \quad \frac{\partial K}{\partial \alpha}=-\frac{\partial G}{\partial \bar{\beta}}, \\
& \bar{\varepsilon}^{+} \Rightarrow \frac{\partial K}{\partial b}=\frac{\partial \bar{F}}{\partial \bar{a}}, \quad \frac{\partial K}{\partial a}=-\frac{\partial \bar{F}}{\partial \bar{b}}, \quad \frac{\partial K}{\partial \bar{\beta}}=\frac{\partial \bar{G}}{\partial \alpha}, \quad \frac{\partial K}{\partial \bar{\alpha}}=-\frac{\partial \bar{G}}{\partial \beta}, \\
& \varepsilon^{-} \Rightarrow \frac{\partial K}{\partial \bar{b}}=-\frac{\partial P}{\partial \bar{a}}, \quad \frac{\partial K}{\partial a}=\frac{\partial P}{\partial b}, \quad \frac{\partial K}{\partial \beta}=-\frac{\partial H}{\partial \alpha}, \quad \frac{\partial K}{\partial \bar{\alpha}}=\frac{\partial H}{\partial \bar{\beta}}, \\
& \bar{\varepsilon}^{-} \Rightarrow \frac{\partial K}{\partial b}=-\frac{\partial \bar{P}}{\partial a}, \quad \frac{\partial K}{\partial \bar{a}}=\frac{\partial \bar{P}}{\partial \bar{b}}, \quad \frac{\partial K}{\partial \bar{\beta}}=-\frac{\partial \bar{H}}{\partial \bar{\alpha}}, \quad \frac{\partial K}{\partial \alpha}=\frac{\partial \bar{H}}{\partial \beta} .
\end{aligned}
$$

Кроме того, возникают условия аналитичности для функций в правой части уравнения (4.4):

$$
\begin{aligned}
& \varepsilon^{+} \Rightarrow \frac{\partial G}{\partial \bar{a}}=0, \frac{\partial G}{\partial \bar{b}}=0, \frac{\partial F}{\partial \alpha}=0, \frac{\partial F}{\partial \beta}=0, \\
& \bar{\varepsilon}^{+} \Rightarrow \frac{\partial \bar{G}}{\partial a}=0, \frac{\partial \bar{G}}{\partial b}=0, \frac{\partial \bar{F}}{\partial \bar{\alpha}}=0, \frac{\partial \bar{F}}{\partial \bar{\beta}}=0, \\
& \varepsilon^{-} \Rightarrow \frac{\partial H}{\partial a}=0, \frac{\partial H}{\partial \bar{b}}=0, \frac{\partial P}{\partial \bar{\alpha}}=0, \frac{\partial P}{\partial \beta}=0, \\
& \bar{\varepsilon}^{-} \Rightarrow \frac{\partial \bar{H}}{\partial \bar{a}}=0, \frac{\partial \bar{H}}{\partial b}=0, \frac{\partial \bar{P}}{\partial \alpha}=0, \frac{\partial \bar{P}}{\partial \bar{\beta}}=0 .
\end{aligned}
$$

Из уравнений (4.18), (4.19) находим условия интегрируемости для потенциала $K$ :

$$
\begin{aligned}
& \frac{\partial^{2} K}{\partial \bar{a} \partial \beta}=0, \frac{\partial^{2} K}{\partial \bar{b} \partial \beta}=0, \frac{\partial^{2} K}{\partial \bar{a} \partial \alpha}=0, \frac{\partial^{2} K}{\partial \bar{b} \partial \alpha}=0, \\
& \frac{\partial^{2} K}{\partial a \partial \bar{\beta}}=0, \frac{\partial^{2} K}{\partial b \partial \bar{\beta}}=0, \quad \frac{\partial^{2} K}{\partial a \partial \bar{\alpha}}=0, \frac{\partial^{2} K}{\partial b \partial \bar{\alpha}}=0, \\
& \frac{\partial^{2} K}{\partial a \partial \beta}=0, \frac{\partial^{2} K}{\partial \bar{b} \partial \bar{\alpha}}=0, \frac{\partial^{2} K}{\partial \bar{a} \partial \bar{\beta}}=0, \frac{\partial^{2} K}{\partial b \partial \alpha}=0 .
\end{aligned}
$$

Кроме того, потенциал $K$ удовлетворяет двум независимым четырехмерным уравнениям Лапласа:

$$
\frac{\partial^{2} K}{\partial a \partial \bar{a}}+\frac{\partial^{2} K}{\partial b \partial \bar{b}}=0, \quad \frac{\partial^{2} K}{\partial \alpha \partial \bar{\alpha}}+\frac{\partial^{2} K}{\partial \beta \partial \bar{\beta}}=0 .
$$

Введем, аналогично предыдушему случаю, дублетные суперполя

$$
a^{\alpha}=(a, \bar{b}), \quad \Gamma^{\alpha}=(\bar{\alpha}, \beta), \quad \bar{a}^{\alpha}=(\bar{a}, b), \quad \bar{\Gamma}^{\alpha}=(\alpha, \bar{\beta})
$$

и перепишем систему уравнений (4.20) в эквивалентном виде:

$$
\begin{aligned}
\frac{\partial^{2} K}{\partial a^{\alpha} \partial \Gamma^{\beta}} & =0, & \frac{\partial^{2} K}{\partial \bar{a}^{\alpha} \partial \bar{\Gamma}^{\beta}} & =0, \\
\varepsilon^{\alpha \beta} \frac{\partial^{2} K}{\partial a^{\alpha} \partial \bar{\Gamma}^{\beta}} & =0, & \varepsilon^{\alpha \beta} \frac{\partial^{2} K}{\partial \bar{a}^{\alpha} \partial \Gamma^{\beta}} & =0
\end{aligned}
$$


(здесь нет суммирования по повторяющимся индексам). Уравнения (4.21), (4.23), (4.24) составляют полную систему дифференциальных связей для функции $K$. Решение уравнений $(4.23),(4.24)$, как и в случае пары несамодуальных $N=(4,4)$ мультиплетов с твистом, представляется в виде суммы двух членов:

$$
K\left(a^{\alpha}, \bar{a}^{\alpha}, \Gamma^{\alpha}, \bar{\Gamma}^{\alpha}\right)=T\left(\Gamma^{\alpha}, \bar{\Gamma}^{\alpha}\right)+h\left(a^{\alpha}, \bar{a}^{\alpha}\right) .
$$

Из уравнений (4.21) следует, что $T$ и $h$ удовлетворяют своим четырехмерным уравнениям Лапласа.

Таким образом, мы показали, что действия обшей сигма-модели как в случае $N=$ $(4,4)$ мультиплетов с твистом, принадлежащих самодуальной паре, так и для несамодуальной пары редуцируются к определенным суммам $N=(4,4)$ суперсимметричных действий отдельных мультиплетов. Другими словами, каждое действие в сумме включает в себя зависимость только от тех $N=(2,2)$ киральных суперполей и киральных суперполей с твистом, которые отвечают определенному фиксированному типу $N=(4,4)$ мультиплета с твистом. Из этих результатов следует, что $N=(2,2)$ киральные суперполя и киральные суперполя с твистом, которые принадлежат разным $N=(4,4)$ мультиплетам и имеют, следовательно, различные трансформационные свойства относительно преобразований скрытой $N=(2,2)$ суперсимметрии, не могут взаимодействовать друг с другом посредством действий сигма-модельного типа.

Анализ, проведенный в $N=(2,2)$ суперпространстве, подтверждает результаты, полученные для разных типов $N=(4,4)$ мультиплетов с твистом в подходе бигармонического суперпространства [21], где редукция общего действия к сумме отдельных действий была доказана для любого числа $N=(4,4)$ мультиплетов с твистом независимо от типа мультиплета. Проведенный здесь анализ также может быть обобщен на случай произвольного числа мультиплетов с тем же окончательным выводом.

4.4. Потенциальные члены. В разделе 3 на примере мультиплета с твистом $\hat{q}^{i a}$ были построены $N=(4,4)$ суперсимметричные потенциальные (массовые) члены в суперпространстве $\mathbb{R}^{(1,1 \mid 2,2)}$. В нашей предыдушей работе [21] было обнаружено, что для мультиплетов с твистом, принадлежащих самодуальной паре, существуют инвариантные смешанные массовые члены. Эти члены имеют такой же вид, как и аналогичные выражения, полученные в работах [1], [4], [13], [14].

Результаты работы [21] могут быть легко переведены на язык $N=(2,2)$ киральных суперполей и киральных суперполей с твистом в суперпространстве $\mathbb{R}^{(1,1 \mid 2,2)}$. Так, например, для самодуальной пары мультиплетов с твистом $\hat{q}^{i} \underline{a}$ и $\hat{q}^{\underline{i}} a$ в качестве подходящего пробного потенциального члена можно выбрать следующую билинейную суперполевую комбинацию:

$$
S_{(4,4)}^{M}=i M \int \mu\left\{l\left(\theta^{+} \theta^{-}\right) \bar{\alpha} \bar{a}+k\left(\bar{\theta}^{+} \bar{\theta}^{-}\right) \alpha a+n\left(\theta^{+} \bar{\theta}^{-}\right) \bar{\beta} \bar{b}+p\left(\bar{\theta}^{+} \theta^{-}\right) \beta b\right\},
$$

а для случая несамодуальной пары $\hat{q}^{i a}$ и $\hat{q}^{i} \underline{a}-$

$$
S_{(4,4)}^{\widetilde{M}}=i \widetilde{M} \int \mu\left\{l^{\prime}\left(\theta^{+} \theta^{-}\right) \bar{a} \bar{A}+k^{\prime}\left(\bar{\theta}^{+} \bar{\theta}^{-}\right) a A+n^{\prime}\left(\theta^{+} \bar{\theta}^{-}\right) \bar{b} \bar{B}+p^{\prime}\left(\bar{\theta}^{+} \theta^{-}\right) b B\right\} .
$$


В уравнениях (4.26), (4.27) $l, l^{\prime}$ и т.д. - некоторые коэффициенты, первоначально произвольные. Они являются аналогами гармонических констант $C^{p, q} \mathrm{c} U(1) \times U(1)$-зарядами $p$ и $q$, фигурируюших в работе [21]. Вычисляя вариацию действия (4.26) относительно преобразований дополнительной $N=(2,2)$ суперсимметрии, находим, что действие (4.26) инвариантно при условии равенства констант:

$$
l=k=n=p \text {. }
$$

В то же время инвариантность потенциального действия (4.27) для несамодуальной пары мультиплетов возможна лишь в случае

$$
l^{\prime}=k^{\prime}=n^{\prime}=p^{\prime}=0
$$

т.е. инвариантные смешанные массовые члены для такой пары не существуют. Аналогичные результаты были получены в работе [21]: из условия $N=(4,4)$ инвариантности пробных потенциальных действий для несамодуальных мультиплетов с твистом следовало равенство нулю гармонических константант, тогда как для самодуальных мультиплетов с твистом было показано, что гармонические константы отличны от нуля и имеют фиксированную зависимость от гармоник.

Отметим также, что наиболее общее $N=(4,4)$ суперсимметричное потенциальное действие вне массовой оболочки представляет собой сумму смешанного массового члена (4.26) с условиями (4.28) и линейных выражений (3.22) для каждого мультиплета с твистом. После исключения вспомогательных полей в полном компонентном действии возникают потеншиальные и массовые члены для физических бозонных полей [21], а также взаимодействия физических фермионов типа Юкавы.

\section{5. ЗАКЛЮЧЕНИЕ}

В настоящей работе изучено описание четырех различных типов $N=(4,4)$ мультиплетов с твистом в двумерном $N=(2,2)$ суперпространстве $\mathbb{R}^{(1,1 \mid 2,2)}$. Показано, что вне массовой оболочки каждый тип $N=(4,4)$ мультиплета с твистом может быть эквивалентным образом представлен парой $N=(2,2)$ кирального суперполя и кирального суперполя с твистом, которые имеют разные трансформационные свойства относительно дополнительной $N=(2,2)$ суперсимметрии (образуюшей вместе с явной $N=(2,2)$ суперсимметрией полную $N=(4,4)$ суперсимметрию). Обшее сигма-модельное действие вне массовой оболочки для любого фиксированного типа $N=(4,4)$ мультиплета с твистом записывается в виде интеграла по суперпространству $\mathbb{R}^{(1,1 \mid 2,2)}$ от вещественной функции $K$, которая зависит от соответствующих пар кирального суперполя и кирального суперполя с твистом и подчиняется определенным дифференциальным связям. Эти связи обеспечивают инвариантность сигма-модельных действий относительно $N=$ $(4,4)$ суперсимметрии и имеют одинаковый вид для всех потенциалов $K$ независимо от того, для каких типов мультиплетов с твистом они определены. Также показано, что требование инвариантности относительно дополнительной $N=(2,2)$ суперсимметрии однозначно фиксирует потенциальные (или массовые) члены $P_{1}, P_{2}$. 
Для более общего случая, когда суперпотенциал $K$ зависит от $N=(2,2)$ киральных суперполей и киральных суперполей с твистом, принадлежаших разным $N=(4,4)$ мультиплетам, показано, что наличие дополнительной $N=(2,2)$ суперсимметрии требует, чтобы общее сигма-модельное действие распадалось в сумму сигма-модельных действий отдельных мультиплетов. Существует единственная возможность устроить взаимодействие между различными типами $N=(4,4)$ мультиплетов с твистом - за счет добавки инвариантных смешанных массовых членов. Эти члены билинейны по киральным суперполям и киральным суперполям с твистом, которые образуют самодуальные пары $N=(4,4)$ мультиплетов. Мультиплеты с твистом, принадлежашие различным самодуальным парам, не могут взаимодействовать меж ду собой ни через сигма-модельные действия, ни через массовые члены.

Суммируя, подчеркнем, что проведенный анализ в рамках стандартного $N=(2,2)$, $2 D$ суперполевого формализма находится в полном соответствии с результатами, полученными в работе [21] для различных типов $N=(4,4)$ мультиплетов с твистом в рам$\operatorname{kax} S U(2) \times S U(2)$ гармонического суперпространства. Развитое здесь $N=(2,2), 2 D$ суперполевое описание для всех типов $N=(4,4)$ мультиплетов с твистом может быть использовано во многих физических и геометрических проблемах, к которым имеют отношение такие мультиплеты.

ПРИЛОЖЕНИЕ А

\section{$N=(2,2), 2 D$ спинорные производные и связи}

Приведем детали обозначений для спинорных производных. Используя квартетные обозначения, определим два различных вида ковариантных производных в левом и правом секторах светового конуса в $2 D$. Эти производные преобразуются как дублеты относительно различных групп автоморфизмов $S U(2)$, объединенных соответственно в группы $S O(4)_{\mathrm{L}}$ и $S O(4)_{\mathrm{R}}$ :

$$
\begin{aligned}
& D_{i \underline{k}}=\left(D_{i \underline{1}}, D_{i \underline{2}}\right) \equiv\left(D_{+i}, \bar{D}_{+i}\right)=\left(D_{1 \underline{k}}, D_{2} \underline{k}\right) \equiv\left(D_{+\underline{k}}, \bar{D}_{+\underline{k}}\right), \\
& D_{a \underline{b}}=\left(D_{a \underline{1}}, D_{a \underline{2}}\right) \equiv\left(D_{-a}, \bar{D}_{-a}\right)=\left(D_{1 \underline{b}}, D_{2 \underline{b}}\right) \equiv\left(D_{-\underline{b}}, \bar{D}_{-\underline{b}}\right) .
\end{aligned}
$$

Ковариантные $N=(2,2)$ спинорные производные $D$ и $d$, соответствуюшие явной и скрытой суперсимметриям, определим следующим образом:

$$
\begin{aligned}
D_{+i}=\left(D_{+}, d_{+}\right)=D_{i \underline{1}}, & \bar{D}_{+}^{i}=\left(\bar{D}_{+}, \bar{d}_{+}\right)=\varepsilon^{i k} \bar{D}_{+k}=\varepsilon^{i k} D_{k \underline{2}} \\
D_{-a}=\left(D_{-}, d_{-}\right)=D_{a \underline{1}}, & \bar{D}_{-}^{a}=\left(\bar{D}_{-}, \bar{d}_{-}\right)=\varepsilon^{a b} \bar{D}_{-b}=\varepsilon^{a b} D_{b \underline{2}} .
\end{aligned}
$$

Связь между $N=(2,2)$ спинорными производными $D, d$ и $S U(2)$-дублетными производными с подчеркнутыми индексами, определенными во вторых строках уравнений (А.1), (А.2), дается соотношениями

$$
\begin{array}{ll}
D_{+\underline{k}}=D_{1 \underline{k}}=\left(D_{+}, \bar{d}_{+}\right), & \bar{D}_{+}^{\underline{i}}=\varepsilon^{\underline{i} \underline{k}} \bar{D}_{\underline{k}}=\left(\bar{D}_{+}, d_{+}\right), \\
D_{-\underline{b}}=D_{1 \underline{b}}=\left(D_{-}, \bar{d}_{-}\right), & \bar{D}_{-}^{\underline{a}}=\varepsilon^{\underline{a}} \underline{\underline{b}} \bar{D}_{\underline{b}}=\left(\bar{D}_{-}, d_{-}\right) .
\end{array}
$$


Спинорные производные в левых координатных секторах суперпространств $\mathbb{R}^{(1,1 \mid 4,4)}$ и $\mathbb{R}^{(1,1 \mid 2,2)}$ имеют следуюший вид:

$$
\begin{aligned}
D_{+i} & =\frac{\partial}{\partial \theta^{+i}}+i \bar{\theta}_{i}^{+} \partial_{++}, & \bar{D}_{+}^{i} & =-\frac{\partial}{\partial \bar{\theta}_{i}^{+}}-i \theta^{+i} \partial_{++}, \\
D_{+} & =\frac{\partial}{\partial \theta^{+}}+i \bar{\theta}^{+} \partial_{++}, & \bar{D}_{+} & =-\frac{\partial}{\partial \bar{\theta}^{+}}-i \theta^{+} \partial_{++}, \\
d_{+} & =\frac{\partial}{\partial \eta^{+}}+i \bar{\eta}^{+} \partial_{++}, & \bar{d}_{+} & =-\frac{\partial}{\partial \bar{\eta}^{+}}-i \eta^{+} \partial_{++} .
\end{aligned}
$$

Для аналогичных выражений в правых секторах имеем

$$
\begin{aligned}
D_{-a} & =\frac{\partial}{\partial \theta^{-a}}+i \bar{\theta}_{a}^{-} \partial_{--}, & \bar{D}_{-}^{a} & =-\frac{\partial}{\partial \bar{\theta}_{a}^{-}}-i \theta^{-a} \partial_{--}, \\
D_{-} & =\frac{\partial}{\partial \theta^{-}}+i \bar{\theta}^{-} \partial_{--}, & \bar{D}_{-} & =-\frac{\partial}{\partial \bar{\theta}^{-}}-i \theta^{-} \partial_{--} \\
d_{-} & =\frac{\partial}{\partial \xi^{-}}+i \bar{\xi}^{-} \partial_{--}, & \bar{d}_{-} & =-\frac{\partial}{\partial \bar{\xi}^{-}}-i \xi^{-} \partial_{--}
\end{aligned}
$$

Генераторы преобразований $N=(4,4)$ суперсимметрии в суперпространстве $\mathbb{R}^{(1,1 \mid 4,4)}$ имеют вид

$$
\begin{aligned}
Q_{+i} & =i \frac{\partial}{\partial \theta^{+i}}+\bar{\theta}_{i}^{+} \partial_{++}, & \bar{Q}_{+}^{i} & =-i \frac{\partial}{\partial \bar{\theta}_{i}^{+}}-\theta^{+i} \partial_{++}, \\
Q_{-a} & =i \frac{\partial}{\partial \theta^{-a}}+\bar{\theta}_{a}^{-} \partial_{--}, & \bar{Q}_{-}^{a} & =-i \frac{\partial}{\partial \bar{\theta}_{a}^{-}}-\theta^{-a} \partial_{--}
\end{aligned}
$$

Для первых компонент в разложении $N=(4,4)$ суперполей по дополнительным грассмановым координатам $\eta$ и $\xi$ в (3.5)-(3.7) мы используем обозначения

$$
\begin{aligned}
& \left.\mathbf{a}\right|_{\eta=\xi=0}=a,\left.\quad \overline{\mathbf{a}}\right|_{\eta=\xi=0}=\bar{a},\left.\quad \mathbf{b}\right|_{\eta=\xi=0}=b,\left.\quad \overline{\mathbf{b}}\right|_{\eta=\xi=0}=\bar{b}, \\
& \left.\mathcal{A}\right|_{\eta=\xi=0}=\alpha,\left.\overline{\mathcal{A}}\right|_{\eta=\xi=0}=\bar{\alpha},\left.\quad \mathcal{B}\right|_{\eta=\xi=0}=\beta,\left.\overline{\mathcal{B}}\right|_{\eta=\xi=0}=\bar{\beta}, \\
& \left.\mathrm{A}\right|_{\eta=\xi=0}=\mathrm{a},\left.\quad \overline{\mathrm{A}}\right|_{\eta=\xi=0}=\overline{\mathrm{a}},\left.\quad \mathrm{B}\right|_{\eta=\xi=0}=\mathrm{b},\left.\quad \overline{\mathrm{B}}\right|_{\eta=\xi=0}=\overline{\mathrm{b}} \text {. }
\end{aligned}
$$

Эти $N=(2,2)$ суперполя удовлетворяют определяющим условиям кирального суперполя и кирального суперполя с твистом:

$$
\begin{array}{rlll}
\bar{D}_{+} a=0, & \bar{D}_{-} a=0, & \bar{D}_{+} b=0, & D_{-} b=0, \\
D_{+} \bar{a}=0, & D_{-} \bar{a}=0, & D_{+} \bar{b}=0, & \bar{D}_{-} \bar{b}=0, \\
\bar{D}_{+} \alpha=0, & \bar{D}_{-} \alpha=0, & \bar{D}_{+} \beta=0, & D_{-} \beta=0, \\
D_{+} \bar{\alpha}=0, & D_{-} \bar{\alpha}=0, & D_{+} \bar{\beta}=0, & \bar{D}_{-} \bar{\beta}=0, \\
\bar{D}_{+} \mathrm{a}=0, & \bar{D}_{-\mathrm{a}}=0, & \bar{D}_{+} \mathrm{b}=0, & D_{-} \mathrm{b}=0, \\
D_{+} \overline{\mathrm{a}}=0, & D_{-} \mathrm{a}=0, & D_{+} \overline{\mathrm{b}}=0, & \bar{D}_{-} \overline{\mathrm{b}}=0 .
\end{array}
$$

Эти условия следуют из $N=(4,4)$ связей $(2.7)-(2.10)$. 


\section{Решение связей для $K$}

\section{ПРИЛОЖЕНИЕ Б}

Получим явное решение условий для суперпотенциала $K$, включающего различные типы $N=(4,4)$ мультиплетов с твистом, принадлежащие к несамодуальной паре. Эти условия имеют вид (4.13), (4.14):

$$
\frac{\partial^{2} K}{\partial a^{\alpha} \partial A^{\beta}}=0, \quad \frac{\partial^{2} K}{\partial \bar{a}^{\alpha} \partial \bar{A}^{\beta}}=0 .
$$

Дублетные обозначения определены в (4.11).

В качестве первого шага частично разрешим (Б.1), вводя комплексные переменные

$$
F_{\beta}\left(\bar{a}^{\alpha}, A^{\alpha}, \bar{A}^{\alpha}\right) \equiv \frac{\partial K}{\partial A^{\beta}}, \quad \bar{F}_{\beta}\left(a^{\alpha}, A^{\alpha}, \bar{A}^{\alpha}\right) \equiv \frac{\partial K}{\partial \bar{A}^{\beta}} .
$$

Из определений $F_{\alpha}$ и $\bar{F}_{\alpha}$ следуют условия интегрируемости

$$
\frac{\partial F_{\alpha}}{\partial \bar{A}^{\beta}}-\frac{\partial \bar{F}_{\beta}}{\partial A^{\alpha}}=0
$$

Действуя на это уравнение оператором $\partial / \partial a^{\rho}$ и используя снова (Б.2), находим

$$
\frac{\partial^{2} \bar{F}_{\beta}}{\partial A^{\alpha} \partial a^{\rho}}=0
$$

из чего следует, что

$$
\frac{\partial \bar{F}_{\alpha}}{\partial A^{\beta}}=G_{\alpha \beta}\left(A^{\rho}, \bar{A}^{\rho}\right)
$$

Аналогично для $F_{\alpha}$ получим

$$
\frac{\partial F_{\alpha}}{\partial \bar{A}^{\beta}}=\bar{G}_{\alpha \beta}\left(A^{\rho}, \bar{A}^{\rho}\right)
$$

Интегрируя уравнения (Б.5) и (Б.6), найдем обшее решение для $F_{\alpha}$ и $\bar{F}_{\alpha}$ :

$$
\begin{aligned}
& F_{\alpha}\left(\bar{a}^{\beta}, A^{\beta}, \bar{A}^{\beta}\right)=f_{\alpha}\left(A^{\beta}, \bar{A}^{\beta}\right)+\bar{G}_{\alpha}\left(A^{\beta}, \bar{a}^{\beta}\right), \\
& \bar{F}_{\alpha}\left(a^{\beta}, A^{\beta}, \bar{A}^{\beta}\right)=\bar{f}_{\alpha}\left(A^{\beta}, \bar{A}^{\beta}\right)+G_{\alpha}\left(\bar{A}^{\beta}, a^{\beta}\right) .
\end{aligned}
$$

Подстановка этих решений в (Б.3) дает

$$
\frac{\partial f_{\alpha}}{\partial \bar{A}^{\beta}}-\frac{\partial \bar{f}_{\beta}}{\partial A^{\alpha}}=0
$$

Решение последних уравнений легко находится и имеет вид

$$
f_{\alpha}=\frac{\partial}{\partial A^{\alpha}} T\left(A^{\beta}, \bar{A}^{\beta}\right), \quad \bar{f}_{\alpha}=\frac{\partial}{\partial \bar{A}^{\alpha}} T\left(A^{\beta}, \bar{A}^{\beta}\right) .
$$


Тогда из уравнений (Б.2), (Б.7), (Б.9) получим

или

$$
\begin{aligned}
& \frac{\partial K}{\partial A^{\alpha}}=F_{\alpha}\left(\bar{a}^{\beta}, A^{\beta}, \bar{A}^{\beta}\right)=\bar{G}_{\alpha}\left(A^{\beta}, \bar{a}^{\beta}\right)+\frac{\partial}{\partial A^{\alpha}} T\left(A^{\beta}, \bar{A}^{\beta}\right), \\
& \frac{\partial K}{\partial \bar{A}^{\alpha}}=\bar{F}_{\alpha}\left(a^{\beta}, A^{\beta}, \bar{A}^{\beta}\right)=G_{\alpha}\left(\bar{A}^{\beta}, a^{\beta}\right)+\frac{\partial}{\partial \bar{A}^{\alpha}} T\left(A^{\beta}, \bar{A}^{\beta}\right),
\end{aligned}
$$

$$
\bar{G}_{\alpha}\left(A^{\beta}, \bar{a}^{\beta}\right)=\frac{\partial}{\partial A^{\alpha}}\{K-T\}, \quad G_{\alpha}\left(\bar{A}^{\beta}, a^{\beta}\right)=\frac{\partial}{\partial \bar{A}^{\alpha}}\{K-T\} .
$$

Эти соотношения предполагают условия интегрируемости

которые, в свою очередь, дают

$$
\frac{\partial \bar{G}_{\alpha}}{\partial A^{\beta}}-\frac{\partial \bar{G}_{\beta}}{\partial A^{\alpha}}=0, \quad \frac{\partial G_{\alpha}}{\partial \bar{A}^{\beta}}-\frac{\partial G_{\beta}}{\partial \bar{A}^{\alpha}}=0,
$$

$$
\bar{G}_{\alpha}=\frac{\partial}{\partial A^{\alpha}} G\left(A^{\beta}, \bar{a}^{\beta}\right), \quad G_{\alpha}=\frac{\partial}{\partial \bar{A}^{\alpha}} \bar{G}\left(\bar{A}^{\beta}, a^{\beta}\right) .
$$

Подстановка (Б.13) в (Б.11) приводит к системе уравнений

$$
\frac{\partial}{\partial A^{\alpha}}\left\{K-T-G\left(A^{\beta}, \bar{a}^{\beta}\right)\right\}=0, \quad \frac{\partial}{\partial \bar{A}^{\alpha}}\left\{K-T-\bar{G}\left(\bar{A}^{\beta}, a^{\beta}\right)\right\}=0,
$$

которая имеет простое решение в виде

$$
\begin{aligned}
& K-T=G\left(A^{\alpha}, \bar{a}^{\alpha}\right)+\Omega\left(\bar{A}^{\alpha}, a^{\alpha}, \bar{a}^{\alpha}\right), \\
& K-T=\bar{G}\left(\bar{A}^{\alpha}, a^{\alpha}\right)+\bar{\Omega}\left(A^{\alpha}, a^{\alpha}, \bar{a}^{\alpha}\right) .
\end{aligned}
$$

Выражая $K-T$ из второго из уравнений (Б.15) и подставляя в первое из уравнений (Б.14), находим

$$
\bar{\Omega}=G\left(A^{\alpha}, \bar{a}^{\alpha}\right)+h\left(a^{\alpha}, \bar{a}^{\alpha}\right) .
$$

Аналогично, подставляя $K-T$ из первого из уравнений (Б.15) во второе из уравнений (Б.14), получим

$$
\Omega=\bar{G}\left(\bar{A}^{\alpha}, a^{\alpha}\right)+h\left(a^{\alpha}, \bar{a}^{\alpha}\right) .
$$

В итоге полное решение уравнений для суперпотенциала $K$ представляется следующей суммой четырех слагаемых:

$$
K\left(A^{\alpha}, \bar{A}^{\alpha}, a^{\alpha}, \bar{a}^{\alpha}\right)=T\left(A^{\alpha}, \bar{A}^{\alpha}\right)+h\left(a^{\alpha}, \bar{a}^{\alpha}\right)+G\left(A^{\alpha}, \bar{a}^{\alpha}\right)+\bar{G}\left(\bar{A}^{\alpha}, a^{\alpha}\right) .
$$

Учитывая определение дублетных величин $A^{\alpha}, a^{\alpha}$ и их комплексно-сопряженных, а также уравнение Лапласа (4.12), приходим к заключению, что первые два члена в (Б.18) соответствуют потенциалам $N=(4,4)$ суперсимметричных сигма-модельных действий для двух независимых мультиплетов с твистом $\hat{q}^{i a}$ и $\hat{q}^{i \underline{a}}$. Последние два члена могут быть устранены обобщенным кэлеровым калибровочным преобразованием в (4.3) с калибровочной функцией $g$. Таким образом, они не дают вклада в $N=(2,2)$ суперполевое действие.

Итак, окончательный результат для потенциала $K$ имеет вид

$$
K\left(A^{\alpha}, \bar{A}^{\alpha}, a^{\alpha}, \bar{a}^{\alpha}\right)=T\left(A^{\alpha}, \bar{A}^{\alpha}\right)+h\left(a^{\alpha}, \bar{a}^{\alpha}\right) .
$$

В случае, когда $N=(2,2)$ киральные суперполя и киральные суперполя с твистом группируются в самодуальную пару $N=(4,4)$ мультиплетов с твистом, рассмотрение проводится аналогично. Полученный результат можно также обобщить на случай с произвольным числом разных $N=(4,4)$ мультиплетов с твистом. 
Благодарности. Авторы благодарны за частичную поддержку INTAS, грант № 0000254, и РФФИ, грант № 03-02-17440. Работа Е.И. также поддержана РФФИ-ДФГ, грант № 02-02-04002, и программой “Гейзенберг-Ландау".

\section{Список литературы}

[1] E. A. Ivanov, S. O. Krivonos. J. Phys. A. 1984. V. 17. P. L671.

[2] S. J. Gates Jr., C. Hull, M. Roček. Nucl. Phys. B. 1984. V. 248. P. 157.

[3] P. S. Howe, G. Papadopoulos. Nucl. Phys. B. 1987. V. 289. P. 264.

[4] E. A. Ivanov, S. O. Krivonos, V. M. Leviant. Nucl. Phys. B. 1988. V. 304. P. 601.

[5] A. Sevrin, W. Troost, A. Van Proeyen. Phys. Lett. B. 1988. V. 208. P. 447; U. Lindström. Phys. Lett. B. 2004. V. 587. P. 216; hep-th/0401100.

[6] A. Sevrin, Ph. Spindel, W. Troost, A. Van Proeyen. Phys. Lett. B. 1988. V. 206. P. 71; Nucl. Phys. B. 1988. V. 308. P. 662.

[7] C. Ahn, M. Roček, K. Schoutens, A. Sevrin. Superspace WZW models and black holes. hep-th/9110035.

[8] E. Kiritsis, C. Kounas, D. Lüst. Int. J. Mod. Phys. A. 1994. V. 9. P. 1361; hep-th/9308124.

[9] M. Roček, K. Schoutens, A. Sevrin. Phys. Lett. B. 1991. V. 265. P. 303.

[10] C. Callan, J. Harvey, A. Strominger. Nucl. Phys. B. 1991. V. 359. P. 611.

[11] T. Buscher, U. Lindstrom, M. Roček. Phys. Lett. B. 1988. V. 202. P. 94; A. Sevrin, J. Troost. Nucl. Phys. B. 1997. V. 492. P. 623; hep-th/9610102; The geometry of supersymmetric sigma-models. hep-th/9610103; M. Grisaru, M. Massar, A. Sevrin, J. Troost. Fortsch. Phys. 1999. V. 47. P. 301; hep-th/9801080; J. Bogaerts, A. Sevrin, S. van der Loo, S. van Gils. Nucl. Phys. B. 1999. V. 562. P. 277; hep-th/9905141.

[12] U. Lindstrom, I. T. Ivanov, M. Roček. Phys. Lett. B. 1994. V. 328. P. 49; hep-th/9401091; I. T. Ivanov, B. Kim, M. Roček. Phys. Lett. B. 1995. V. 343. P. 133; hep-th/9406063.

[13] O. Gorovoy, E. Ivanov. Nucl. Phys. B. 1992. V. 381. P. 394.

[14] S. J. Gates Jr. Phys. Lett. B. 1994. V. 338. P. 31; hep-th/9410149.

[15] A. Galperin, E. Ivanov, S. Kalitzin, V. Ogievetsky, E. Sokatchev. Class. Q. Grav. 1984. V. 1. P. 469; A. Galperin, E. Ivanov, V. Ogievetsky, E. Sokatchev. Harmonic Superspace. Cambridge: Cambridge Univ. Press, 2001.

[16] E. Ivanov, A. Sutulin. Nucl. Phys. B. 1994. V. 432. P. 246; 1997. V. 483. P. 531 (Erratum); hep-th/9404098.

[17] E. Ivanov, A. Sutulin. Class. Q. Grav. 1997. V. 14. P. 843; hep-th/9604186; S. Bellucci, E. Ivanov. Nucl. Phys. B. 2000. V. 587. P. 445; hep-th/0003154.

[18] E. A. Ivanov. Phys. Rev. D. 1996. V. 53. P. 2201; hep-th/9502073; Phys. Lett. B. 1995. V. 356. P. 239; hep-th/9504070.

[19] E. A. Ivanov. Nucl. Phys. B (Proc. Suppl.). 1997. V. 52A. P. 354.

[20] S. J. Gates Jr., S. V. Ketov. Phys. Lett. B. 1998. V. 418. P. 111; P. 119; hep-th/9504077.

[21] E. Ivanov, A. Sutulin. Phys. Rev. D. 2004. V. 70. P. 045022; hep-th/0403130.

[22] S. J. Gates Jr., L. Rana. Phys. Lett. B. 1995. V. 345. P. 233; hep-th/9411091; R. Dhanawittayapol, S. J. Gates Jr., L. Rana. Phys. Lett. B. 1996. V. 389. P. 264; hep-th/9606108.

[23] W. Siegel. Class. Q. Grav. 1985. V. 2. P. L41. 